\title{
MATRIX INVARIANTS AND COMPLETE INTERSECTIONS
}

\author{
by LIEVEN LE BRUYN and YASUO TERANISHI
}

(Received 13 February, 1989)

Consider the vector space of $m$-tuples of $n$ by $n$ matrices

$$
X_{m, n}=M_{n}(\mathbb{C}) \oplus \ldots \oplus M_{n}(\mathbb{C})
$$

The linear group $\mathrm{GL}_{n}(\mathbb{C})$ acts on $X_{m, n}$ by simultaneous conjugation. The corresponding ring of polynomial invariants

$$
\mathbb{C}\left[X_{m, n}\right]^{\mathrm{GL}_{n}(\mathbb{C})}
$$

will be denoted by $C(n, m)$ and is called the ring of matrix invariants of $m$-tuples of $n$ by $n$ matrices. C. Procesi has shown in [8] that $C(n, m)$ is generated by traces of products of the corresponding generic matrices and, as such, coincides with the center of the trace ring of $m$ generic $n$ by $n$ matrices $R(n, m)$, which is also the ring of equivariant maps from $X_{m, n}$ to $M_{n}(\mathbb{C})$.

Apart from this general result, very little is known about the explicit structure of $C(n, m)$. In [7], it is shown that $C(2,2)$ is the polynomial algebra

$$
\mathbb{C}\left[\operatorname{Tr}\left(X_{1}\right), \operatorname{Tr}\left(X_{2}\right), \operatorname{Det}\left(X_{1}\right), \operatorname{Det}\left(X_{2}\right), \operatorname{Tr}\left(X_{1} X_{2}\right)\right] \text {. }
$$

The structure of $C(2,3)$ was determined by Formanek [0], see also [3] or [10]. Consider the polynomial algebra

$$
\mathbb{C}\left[\operatorname{Tr}\left(X_{1}\right), \operatorname{Tr}\left(X_{2}\right), \operatorname{Tr}\left(X_{3}\right), D\left(X_{1}\right), D\left(X_{2}\right), D\left(X_{3}\right), \operatorname{Tr}\left(X_{1} X_{2}\right), \operatorname{Tr}\left(X_{2} X_{3}\right), \operatorname{Tr}\left(X_{3} X_{1}\right)\right] ;
$$

then $C(2,3)$ is a free module over this algebra of rank 2 generated by 1 and $\operatorname{Tr}\left(X_{1} X_{2} X_{3}\right)$. Moreover, $\operatorname{Tr}\left(X_{1} X_{2} X_{3}\right)$ satisfies the quadratic equation

where

$$
X^{2}-A X+B=O,
$$

$$
\begin{aligned}
& A=\operatorname{Tr}\left(X_{1}\right) \operatorname{Tr}\left(X_{2} X_{3}\right)+\operatorname{Tr}\left(X_{2}\right) \operatorname{Tr}\left(X_{1} X_{3}\right)+\operatorname{Tr}\left(X_{3}\right) \operatorname{Tr}\left(X_{1} X_{2}\right)-\operatorname{Tr}\left(X_{1}\right) \operatorname{Tr}\left(X_{2}\right) \operatorname{Tr}\left(X_{3}\right), \\
& B= D\left(X_{1}\right) \operatorname{Tr}\left(X_{2} X_{3}\right)^{2}+D\left(X_{2}\right) \operatorname{Tr}\left(X_{1} X_{3}\right)^{2}+D\left(X_{3}\right) \operatorname{Tr}\left(X_{1} X_{2}\right)^{2} \\
&-\operatorname{Tr}\left(X_{1}\right) \operatorname{Tr}\left(X_{2}\right) \operatorname{Tr}\left(X_{1} X_{2}\right) D\left(X_{3}\right)-\operatorname{Tr}\left(X_{1}\right) \operatorname{Tr}\left(X_{3}\right) \operatorname{Tr}\left(X_{1} X_{3}\right) D\left(X_{2}\right) \\
&-\operatorname{Tr}\left(X_{2}\right) \operatorname{Tr}\left(X_{3}\right) \operatorname{Tr}\left(X_{2} X_{3}\right) D\left(X_{1}\right) \\
&+\operatorname{Tr}\left(X_{1}\right)^{2} D\left(X_{2}\right) D\left(X_{3}\right)+\operatorname{Tr}\left(X_{2}\right)^{2} D\left(X_{1}\right) D\left(X_{3}\right) \\
&+\operatorname{Tr}\left(X_{3}\right)^{2} D\left(X_{1}\right) D\left(X_{2}\right) \\
&-4 D\left(X_{1}\right) D\left(X_{2}\right) D\left(X_{3}\right)+\operatorname{Tr}\left(X_{1} X_{2}\right) \operatorname{Tr}\left(X_{1} X_{2}\right) \operatorname{Tr}\left(X_{2} X_{3}\right) .
\end{aligned}
$$

In general, $C(2, m)$ is a polynomial algebra in $m$ variables over the center of the generic Clifford algebra for $m$-ary quadratic forms of degree at most 4, see [2], and it can be expressed in terms of $\mathrm{SO}_{3}(\mathbb{C})$-invariants, see [9].

The structure of $C(3,2)$ was determined in [10] and implicitly in [4]. Consider the

Glasgow Math. J. 32 (1990) 227-229. 
polynomial algebra

$\mathbb{C}\left[\operatorname{Tr}\left(X_{1}\right), \operatorname{Tr}\left(X_{1}^{2}\right), \operatorname{Tr}\left(X_{1}^{3}\right), \operatorname{Tr}\left(X_{2}\right), \operatorname{Tr}\left(X_{2}^{2}\right), \operatorname{Tr}\left(X_{2}^{3}\right), \operatorname{Tr}\left(X_{1} X_{2}\right)\right.$,

$$
\left.\operatorname{Tr}\left(X_{1} X_{2}^{2}\right), \operatorname{Tr}\left(X_{1}^{2} X_{2}\right), \operatorname{Tr}\left(X_{1}^{2} X_{2}^{2}\right)\right]
$$

$C(3,2)$ is a free module of rank 2 over this algebra generated by 1 and $\operatorname{Tr}\left(X_{1} X_{2} X_{1}^{2} X_{2}^{2}\right)$. Apart from these results only $C(4,2)$ is known, see $[10]$.

What is known about the homological properties of $C(n, m)$ ? In view of the Hochster-Roberts results, $C(n, m)$ is a Cohen-Macaulay algebra and, because it is the ring of invariants of the simple group $\mathrm{PGL}_{n}(\mathbb{C})$, it is a unique factorization domain and hence Gorenstein, see for example [1]. In [5], it is shown that $C(n, m)$ is never regular except when $(m, n)=(2,2)$, which is, as we have seen above, a polynomial algebra. Recall that an algebra $\mathbb{C}\left[X_{1}, \ldots, X_{k}\right] / I$ is said to be a complete intersection if the height of $I$ coincides with the minimal number of generators of $I$. It follows from the above explicit descriptions that $C(2,3)$ and $C(3,2)$ are hypersurfaces and hence complete intersections. The main result of this note will assert that there are no others.

Before we come to the proof, we recall some results of [5]. Let $V_{m, n}$ be the variety corresponding to $C(n, m)$; then it is well known that $V_{m, n}$ parametrizes the isomorphism classes of semi-simple $n$-dimensional representations of $\mathbb{C}\left\langle X_{1}, \ldots, X_{m}\right\rangle$. A point $\xi \in V_{m, n}$ is said to be of representation type $\tau=\left(e_{1}, k_{1} ; \ldots ; e_{r}, k_{r}\right)$ if the corresponding isomorphism class of semi-simple representations is built from $r$ distinct simple components of dimensions $k_{i}$ occurring with multiplicity $e_{i}$. If $\tau$ is such a representation type then $V_{m, n}(\tau)$ is defined to be the subset of $V_{m, n}$ consisting of all points of representation type $\tau$. In [5], it is shown that the sets $V_{m, n}(\tau)$ form a finite stratification into locally closed smooth subvarieties. Moreover $V_{m, n}(\tau)$ lies in the closure of $V_{m, n}\left(\tau^{\prime}\right)$ if and only if $\tau$ is a degeneration of $\tau^{\prime}$. If $\xi \in V_{m, n}$ is of representation type $\left(e_{1}, k_{1} ; \ldots ; l_{r}, k_{r}\right)$ then one forms the quiver $\Delta_{\xi}$ consisting of $r$ vertices $\left(x_{1}, \ldots, x_{r}\right)$ and $(m-1) k_{i}^{2}+1$ loops in vertex $x_{1}$ and $(m-1) k_{i} k_{j}$ directed edges from $x_{i}$ to $x_{j}$. Let $d_{\xi}$ be the dimension vector $\left(e_{1}, \ldots, e_{r}\right)$. Then it is proved in [5] that the étale locale structure of $V_{m, n}$ near $\xi$ is that of the variety $V\left(\Delta_{\xi}, d_{\xi}\right)$ of semi-simple representations of the quiver $\Delta_{\xi}$ of dimension vector $d_{\xi}$ near the origin. Moreover, the coordinate ring of $V\left(\Delta_{\xi}, d_{\xi}\right)$ is generated by traces of oriented cycles in the quiver $\Delta_{\xi}$, see [6].

Let $V$ be the variety corresponding to the algebra $\mathbb{C}\left[x_{1}, \ldots, x_{k}\right] / I$; then $V$ is said to be locally a complete intersection in $v$ if $\mathbb{I C}\left[x_{1}, \ldots, x_{k}\right]_{m}$ is generated by a regular sequence of length the height of $I$ for all maximal ideals $m$ lying over $v$. It is clear that the subset of all points $v \in V$ such that $V$ is locally a complete intersection in $v$ forms an open subvariety $V^{\text {c.i }}$. We are now in a position to state the main result.

THEOREM. If $(m, n) \neq(2,2),(2,3)$ or $(3,2)$ then the locally complete intersection locus $V_{m, n}^{\mathrm{ci}}$ coincides with the open subvariety of regular points $V_{m, n}^{\mathrm{reg}}=V_{m, n}[(1, n)]$.

Proof. Since being locally a complete intersection can be expressed in homological terms, it is preserved under étale extensions; so we only need to study the étale local structure of $V_{m, n}$ near a point $\xi$. Suppose $V_{m, n}^{c . i}$ is strictly larger than $V_{m, n}[(1, n)]$, which is precisely the nonsingular locus when $(m, n) \neq(2,2)$, see [5, Theorem II.3.4]. Then, by the stratification result mentioned before, $V_{m, n}^{\text {c.i }}$, must contain a point $\xi$ corresponding to a semi-simple representation having two distinct simple components, i.e. $\xi$ is of type 
$(1, a ; 1, b)$ with $a+b=n$. The corresponding quiver $\Delta_{\xi}$ is

$$
(m-1) a^{2}+1 \bigcirc \overbrace{1}^{\longrightarrow} \underset{(m-1) a b}{(m-1) a b} \bigcirc(m-1) b^{2}+1
$$

and the dimension vector $d_{\xi}=(1,1)$. The coordinate ring of $V\left(\Delta_{\xi}, d_{\xi}\right)$ is then a polynomial algebra in $(m-1)\left(a^{2}+b^{2}\right)+2$ variables over

$$
\mathbb{C}[W]=\mathbb{C}\left[t_{i j}: 1 \leq i, j \leq(m-1) a b\right] / I_{2},
$$

where $I_{2}$ is the ideal generated by the determinants of all 2 by 2 minors of the generic matrix $\left(t_{i j}\right)_{i, j}$. It is well known that $W$ is a complete intersection in the origin if and only if $(m-1) a b=2$. By Etale decent, this finishes the proof.

In view of the explicit descriptions of $C(2,2), C(2,3)$ and $C(3,2)$ given before, and the above theorem, we obtain the following corollary immediately.

COROLlaRY. The ring of matrix invariants $C(m, n)$ is a complete intersection if and only if $(m, n)=(2,2),(2,3)$ or $(3,2)$. In particular, it is a complete intersection if and only if the corresponding ring of equivariant maps $R(n, m)$ is of finite global dimension.

The last statement follows from [5].

\section{REFERENCES}

0. E. Formanek, Invariants and the ring of generic matrices, J. Algebra 89 (1984), 178-223.

1. L. le Bruyn, The Artin-Schofield theorem and some applications, Comm. Algebra 14 (1986), 1439-1455.

2. L. le Bruyn, Trace rings of generic 2 by 2 matrices, Mem. Amer. Math. Soc. 363 (1987).

3. L. le Bruyn and M. van den Bergh, An explicit description of $\mathbb{T}_{3,2}$, Ring theory (Ed. F. M. J. van Oystaeyen), Lecture Notes in Mathematics No. 1197 (Springer, 1986), 109-113.

4. L. le Bruyn and M. van den Bergh, Regularity of trace rings of generic matrices, $J$. Algebra 117 (1988), 19-29.

5. L. le Bruyn and C. Procesi, Etale local structure of matrix invariants and concomitants, Algebraic groups, Utrecht 1986 (Ed. A. M. Cohen, W. H. Hesselink, W. L. J. van der Kallen and J. R. Strooker), Lecture Notes in Mathematics 1271 (Springer, 1987), 143-175.

6. L. le Bruyn and C. Procesi, Semi-simple representations of quivers, Trans. Amer. Math. Soc. to appear.

7. C. Procesi, Rings with polynomial identities (Marcel Dekker, 1973).

8. C. Procesi, Invariant theory of $n \times n$ matrices, Adv. in Math. 19 (1976), 306-381.

9. C. Procesi, Computing with $2 \times 2$ matrices, J. Algebra 87 (1984), 342-359.

10. Y. Teranishi, The ring of invariants of matrices, Nagoya Math. J. 104 (1986), 149-161.

UNIVERSITY OF ANTWERP

UIA-NFWO
UNIVERSITY OF MANNHEIM FRG

and

UNIVERSITY OF NAGOYA

JAPAN 